\title{
Influence de la nature et de la proportion du fourrage et du concentré sur le transit digestif ruminal de la chêvre à l'entretien
}

\author{
H Archimède 1, C Poncet 2, D Sauvant ${ }^{1}$, J Hervieu ${ }^{1}$, \\ 1 INRA - INA-PG, station de Nutrition et Alimentation, 16, rue Claude-Bernard, \\ 75231 Paris Cedex 05; \\ ${ }^{2}$ CRZV - INRA, station de Nutrition des herbivores, Clermont-Ferrand, Theix, 63122 Ceyrat, France
}

Summary - Effect of nature and proportion of forage and concentrate in the diet, on the rate of passage of rumen digesta in the goat fed at near maintenance. The decreasing rate of $\mathrm{Yb}$ and $\mathrm{Cr}$-EDTA in the duodenum $(\mathrm{K})$ after their continuous infusion in the rumen were calculated in goats fed at 1.1 maintenance with 14 mixed diets differing in the nature and the proportion of forage (maize stover, lucerne hay) and concentrate (rich in degradable cell wall, or rapidly degradable starch, or slowly degradable strarch). $K$ increased with the proportion of concentrate but was not affected by its nature. $\mathrm{K}$ was also higher for lucerne hay vs maize stover.

Afin de préciser l'influence de la nature des aliments de la ration et de la proportion de concentré sur le transit digestif, le taux de renouvellement par passage des phases liquide et solide du contenu du rumen a été estimé pour 14 rations complètes comprenant du foin de luzerne (FL) ou des cannes de maïs (CM) associés à 30 ou $60 \%$ de 3 types d'aliments concentrés.

Matériel et méthodes - Les aliments concentrés ont été formulés de manière à posséder des proportions importantes d'ingrédients riches en amidon à dégradation rapide (orge, avoine, blé) ou lente (maïs, sorgho) ou à paroi digestible (pulpe de betteraves, coques de soja). Ces concentrés étaient broyés à la grille de $1 \mathrm{~mm}$, tandis que le FL et les CM étaient hachés en brins de 5 et $2 \mathrm{~cm}$ respectivement. Chacune des 14 rations a été étudiée sur 4 chèvres au moins ( 57 mesures en tout). Les animaux étaient alimentés de manière à excéder de $10 \%$ leur besoin d'entretien. Des doses journalières de $200 \mathrm{mg}$ de chrome $(\mathrm{Cr})$ ou d'Ytterbium $(\mathrm{Yb})$, sous la forme de $130 \mathrm{ml}$ de solution, ont été infusées en continu dans le rumen pendant $10 \mathrm{j}$. Après l'arrêt d'infusion, des échantillons de contenu duodénal ont été prélevés au temps $0,1,3,5,7,9,11,13,15,19,23$, $27,29,31,33 \mathrm{~h}$. Les cinétiques de décroissance des concentrations du Cr et de I'Yb ont été ajustées par un modèle exponentiel à 1 compartiment. Les variations des 57 taux de renouvellement $K$ des marqueurs $\left(K_{c r}\right.$ et $\left.K_{y b}\right)$ ainsi obtenus ont été analysées en fonction de la nature du fourrage ( $\alpha \mathrm{i})$ et du concentré ( $\beta \mathrm{j}$ ), de la proportion de concentré $\left(\mathrm{C}_{i j}, \mathrm{C}_{\mathrm{ii}}{ }^{2}\right)$ et du niveau de matière sèche ingérée (MSlij) selon le modèle :

$$
\begin{aligned}
K_{C_{r}} \text { ou } K_{Y b} & =\mu+\alpha i+\beta j+a C_{i j}+b C_{i j}^{2} \\
& +c\left(\alpha i \cdot C_{i j}\right)+d M S S_{i j}+e_{i j k}
\end{aligned}
$$

dans lequel $e_{i j k}$ est l'écart de l'observation $k$ par rapport au modèle.

Résultats et discussion - Les ajustements de 57 cinétiques de concentration de marqueurs aboutissent à des valeurs moyennes (écart type, ET) et $\mathrm{K}$ de 5,32 $(1,95) \% h^{-1}$ pour les cinétiques l'Yb et de $6,79(2,83) \% \mathrm{~h}^{-1}$ pour le $\mathrm{Cr}$. Les valeurs extrêmes de $R^{2}$ des ajustements sont égaux à 0,81-0,99 pour l'Yb et 0,83-0,99 pour le $\mathrm{Cr}$. Les valeurs de $\mathrm{K}_{\mathrm{Yb}}$ et $\mathrm{K}_{\mathrm{Cr}}$ sont positivement corrélées $\left(K_{\mathrm{Yb}}=0,49 \mathrm{~K}_{\mathrm{Cr}}+\right.$ $2,08, \mathrm{R}^{2}=0,58 P<0,001$ ), ce qui traduit le rôle de véhicule joué par l'eau à l'égard des petites particules qui sont préférentiellement marquées par $\mathrm{Yb}$. La nature du concentré, le niveau de MSi $(1006 \pm 174$ $\mathrm{g} / \mathrm{j})$, l'effet quadratique du niveau de 
concentré et l'interaction fourrage concentré du modèle n'ont pas eu d'effet significatif sur les valeurs de $K$. Les équations d'ajustement

$$
\begin{aligned}
K Y b & =2,66(0,43)+1,66(0,37) \\
& \times F+4,65(0,87) \times C \\
\left(R^{2}\right. & \left.=0,46, E T R=1,39 \% h^{-1}\right) \\
K C r & =3,20(0,76)+2,36(0,65) \\
& \times F+5,67(1,54) \times C \\
\left(R^{2}\right. & \left.=0,32, E T R=2,44 \% h^{-1}\right)
\end{aligned}
$$

dans lesquelles $F=1$ avec les rations $F L$ et $F=0$ avec les rations $C M$, indiquent que les taux de renouvellement de l'Yb et du $\mathrm{Cr}$ avec les rations contenant $\mathrm{FL}$ sont supérieurs respectivement de 1,66 et $2,36 \% \mathrm{~h}^{-1}$ à ceux observés avec les rations $\mathrm{CM}$. Les rations contenant des $\mathrm{CM}$, plus riches en fibres (ADF $=42$ vs $35 \%$ MS) et moins digestibles ( $\mathrm{dMO}=54$ vs $64 \%$ ) que le $\mathrm{FL}$, sont plus favorables à l'accumulation dans le rumen d'une masse alimentaire plus importante, réduisant le taux d'évacuation des particules alimentaires et de l'eau. Les volumes estimés des contenus ruminaux sont en effet de $11,3( \pm 3,9)$ I pour les $\mathrm{CM}$ contre 7,8 $( \pm 3,5)$ I pour le $F L$. L'écart $\mathrm{K}_{\mathrm{Cr}}-\mathrm{K}_{\mathrm{Yb}}$ est supérieur avec les rations $\mathrm{FL}$, en raison probablement d'une plus grande différenciation entre les phases solide et liquide dans le contenu ruminal. Ce résultat pourrait tra- duire le hachage plus grossier du FL que des CM.

$\mathrm{K}_{\mathrm{Yb}}$ et $\mathrm{D}_{\mathrm{Cr}}$ augmentent avec le pourcentage de concentré, comme l'ont observé Kennedy et Bunting (1992) pour du fourrage marqué avec $\mathrm{Yb}$. II est connu qu'au sein d'une même ration la fraction alimentaire provenant du concentré transite plus rapidement (Najar et al, 1988). Cependant, le marquage préférentiel des petites particules, qui sont celles qui transitent plus rapidement, peut contribuer à expliquer nos résultats. D'autre part, différents auteurs, dont Poncet et al (1987), ont observé qu'une ration plus riche en concentré transitait globalement plus lentement, peut-être par altération des phénomènes moteurs.

En conclusion, chez la chèvre nourrie au voisinage de l'entretien, le taux de renouvellement des contenus du rumen a varié avec la nature du fourrage et augmenté avec la proportion d'aliment concentré. Cependant, ce dernier aspect demande à être confirmé avec d'autres méthodologie.

Kennedy DW, Bunting LD (1992) An Feed Sci Technol 36, 91-100

Najar T, Giger S, Poncet C, Sauvant D (1988) Reprod Nutr Dev 28, 133-114

Poncet C, Gonzalez J, Michalet-Doreau B (1987) Reprod Nutr Dev 27, 257-258 